\title{
Fundamental Limits of Caching
}

\author{
Mohammad Ali Maddah-Ali and Urs Niesen
}

\begin{abstract}
Caching is a technique to reduce peak traffic rates by prefetching popular content into memories at the end users. Conventionally, these memories are used to deliver requested content in part from a locally cached copy rather than through the network. The gain offered by this approach, which we term local caching gain, depends on the local cache size (i.e, the memory available at each individual user). In this paper, we introduce and exploit a second, global, caching gain not utilized by conventional caching schemes. This gain depends on the aggregate global cache size (i.e., the cumulative memory available at all users), even though there is no cooperation among the users.

To evaluate and isolate these two gains, we introduce an information-theoretic formulation of the caching problem focusing on its basic structure. For this setting, we propose a novel coded caching scheme that exploits both local and global caching gains, leading to a multiplicative improvement in the peak rate compared to previously known schemes. In particular, the improvement can be on the order of the number of users in the network. Moreover, we argue that the performance of the proposed scheme is within a constant factor of the information-theoretic optimum for all values of the problem parameters.
\end{abstract}

\section{INTRODUCTION}

The high temporal variability of network traffic results in communication systems that are congested during peak-traffic times and underutilized during off-peak times. One approach to reduce peak traffic is to take advantage of memories distributed across the network (at end users, servers, routers, ...) to duplicate content. This duplication of content, called content placement or caching, is performed during off-peak hours when network resources are abundant. During peak hours, when network resources are scarce, user requests can then be served from these caches, reducing network congestion. In this manner, caching effectively allows to shift traffic from peak to off-peak hours, thereby smoothing out traffic variability and reducing congestion.

From the above discussion, we see that the caching problem consists of two distinct phases. The first phase is the placement phase, which is based solely on the statistics of the user demands. In this phase, the network is not congested, and the main limitation is the size of the cache memories. The second phase is the delivery phase, which is performed once the actual demands of the users have been revealed. In this phase, the network is congested, and the main limitation is the rate required to serve the requested content.

Various versions of this problem have been studied, with the focus being mainly on exploiting the history or statistics of the user demands [1]-[7]. In these papers, the operation of the delivery phase is fixed to consist of simple orthogonal unicast or multicast transmissions. Assuming this method of delivery, the content placement is then optimized. The gain of caching in this approach results from making popular content available locally. In another line of research, the objective is to optimize the delivery phase for fixed known cache contents and for specific demands [8], [9] (see also the discussion in Section VIII-A).

As pointed out above, the gain from traditional uncoded caching approaches derives from making content available locally: if a user requests some content that is stored in its cache, this request can be served from its local memory. We hence call this the local caching gain. This gain is relevant if the local cache memory is large enough such that a sizable fraction of the total (popular) content can be stored locally. On the other hand, if the size of the local caches is small compared to the total amount of content, then this gain is insignificant.

This paper was presented in part at the International Symposium on Information Theory, July 2013.

The authors are with Bell Labs, Alcatel-Lucent. Emails: \{mohammadali.maddah-ali, urs.niesen\}@alcatel-lucent.com 
In this paper, we propose a novel coded caching approach that, in addition to the local caching gain, is able to achieve a global caching gain. This gain derives from jointly optimizing both the placement and delivery phases, ensuring that in the delivery phase several different demands can be satisfied with a single coded multicast transmission. Since the content placement is performed without knowledge of the actual demands, in order to achieve this gain the placement phase must be carefully designed such that these multicasting opportunities are created simultaneously for all possible requests in the delivery phase. We show that this global caching gain is relevant if the aggregate global cache size is large enough compared to the total amount of content. Thus, even though the caches cannot cooperate, the sum of the cache sizes becomes an important system parameter.

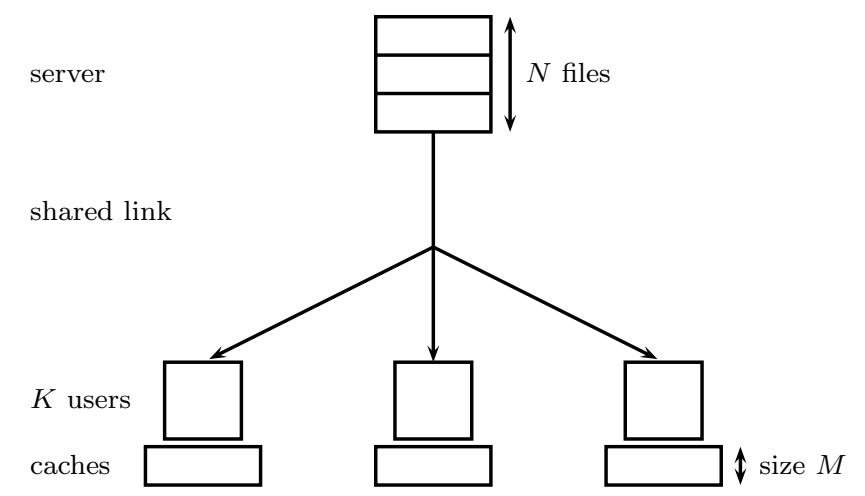

Fig. 1. Caching system considered in this paper. A server containing $N$ files of size $F$ bits each is connected through a shared link to $K$ users each with an isolated cache of size $M F$ bits. The goal is to design the placement phase and the delivery phase such that the peak rate (i.e. the load normalized by the file size) of the shared bottleneck link is minimized. In the figure, $N=K=3$ and $M=1$.

To formally analyze the performance of the proposed coded caching approach, and in order to evaluate and isolate these two gains, we introduce a new, information-theoretic formulation of the caching problem focusing on its basic structure. In our setting, depicted in Fig. 1, $K$ users are connected to a server through a shared, error-free link. The server has a database of $N$ files of equal size. Each of the users has access to a cache memory big enough to store $M$ of the files. During the placement phase, the caches are filled as a function of the database. During the delivery phase, each user may ask for any one of the $N$ possible files. The objective is to design the placement and delivery phases such that the load of the shared link in the delivery phase is minimized. For simplicity, we restrict the discussion in the introduction section to the most relevant case, in which the number of files $N$ is larger than or equal to the number of users $K$. The main results are presented later for the general case.

In this setting, the rate, i.e. the load of the shared link normalized by the file size, in the delivery phase of conventional uncoded caching schemes is

$$
K \cdot(1-M / N) \text {. }
$$

Here $K$ is the rate without caching, and $1-M / N$ is the local caching gain. In contrast, the coded caching scheme proposed in this paper attains a rate of

$$
K \cdot(1-M / N) \cdot \frac{1}{1+K M / N}
$$

Thus, in addition to the local caching gain of $1-M / N$, coded caching also achieves a global caching gain of $\frac{1}{1+K M / N}$. Both of these gains indicate the multiplicative reduction in rate of the shared link, so that a smaller factor means a larger rate reduction.

Observe that the local caching gain depends on the normalized local cache size $M / N$ and is relevant only if the cache size $M$ is on the order of the number of files $N$. On the other hand, the global caching gain depends on the normalized cumulative cache size $K M / N$ and is relevant whenever the cumulative cache size $K M$ is on the order of (or larger than) the number of files $N$. 
By deriving fundamental lower bounds on the rate required in the delivery phase, we show that the rate of the proposed coded caching scheme is within a factor 12 of the information-theoretic optimum for all values of $N, K$, and $M$.

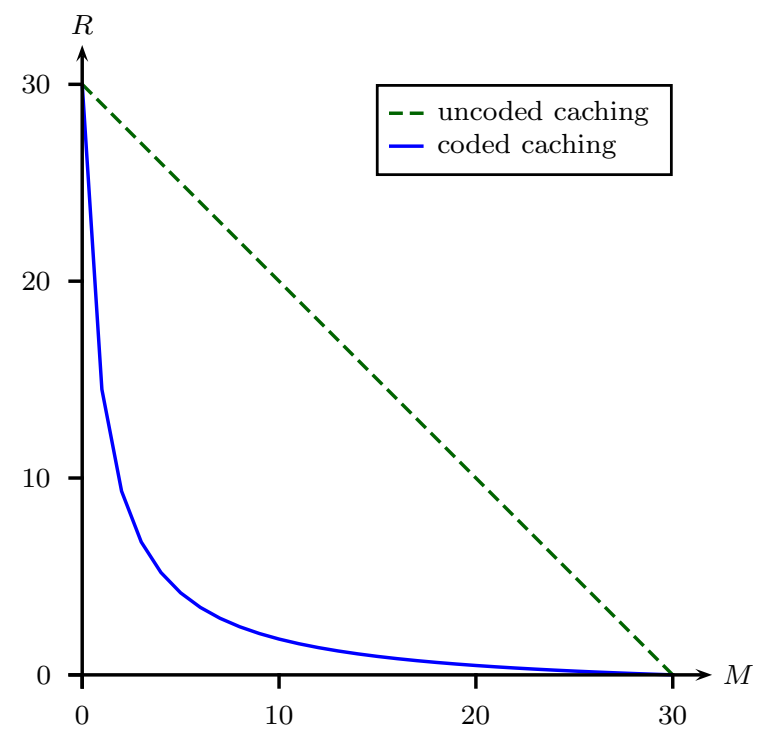

Fig. 2. Rate $R$ required in the delivery phase as a function of memory size $M$ for $N=30$ files and $K=30$ users. The figure compares the performance of the proposed coded caching scheme to that of conventional uncoded caching.

To obtain some intuition for the effect of these two gains, let us compare the rates of the conventional uncoded scheme (achieving only the local gain) versus the proposed coded scheme (achieving both the local and global gains) for a system with $N=30$ files and $K=30$ users as shown in Fig. 2, When each user has a cache memory large enough to store $M=10$ files, the rate of the uncoded caching scheme corresponds to sending 20 files over the shared link, while the proposed coded caching scheme achieves a rate corresponding to sending only 1.8 files: a reduction by a factor 11 in rate.

The remainder of this paper is organized as follows. Section [II formally introduces our informationtheoretic formulation of the caching problem. Section III presents main results, which are illustrated with examples in Section [V] Sections V-VII contain proofs. Section VIII discusses some follow-up results and directions for future research.

\section{Problem Setting}

Before formally introducing the problem in Section $\amalg$ II-B, we start with an informal description in Section II-A

\section{A. Informal Problem Description}

We consider a system with one server connected through a shared, error-free link to $K$ users, as shown in Fig. 11. The server has access to a database of $N$ files $W_{1}, \ldots, W_{N}$ each of size $F$ bits. Each user $k$ has an isolated cache memory $Z_{k}$ of size $M F$ bits for some real number $M \in[0, N]$.

The system operates in two phases: a placement phase and a delivery phase. In the placement phase, the users are given access to the entire database $W_{1}, \ldots, W_{N}$ of files. Each user $k$ is then able to fill the content of its cache $Z_{k}$ using the database. In the delivery phase, only the server has access to the database of files. Each user $k$ requests one of the files $W_{d_{k}}$ in the database. The server is informed of these requests and proceeds by transmitting a signal $X_{\left(d_{1}, \ldots, d_{k}\right)}$ of size $R F$ bits over the shared link for some fixed real number $R$. The quantities $R F$ and $R$ are referred to as the load and the rate of the shared link, respectively. Using the content $Z_{k}$ of its cache and the signal $X_{\left(d_{1}, \ldots, d_{k}\right)}$ received over the shared link, each user $k$ aims to reconstruct its requested file $W_{d_{k}}$. 
A memory-rate pair $(M, R)$ is achievable for requests $d_{1}, \ldots, d_{K}$ if every user $k$ is able to recover its desired file $W_{d_{k}}$ (with high probability for $F$ large enough). A memory-rate pair $(M, R)$ is said to be achievable if this pair is achievable for every possible request $d_{1}, \ldots, d_{K}$ in the delivery phase. Finally, we denote by $R^{\star}(M)$ the smallest rate $R$ such that $(M, R)$ is achievable. The function $R^{\star}(M)$ describes the memory-rate tradeoff for the caching problem. The aim of this paper is to characterize this memory-rate tradeoff. In other words, we aim to find the minimum rate of communication over the shared link at which all possible demand tuples can be satisfied.

We illustrate these definitions with the example of the caching strategy employed by conventional uncoded caching systems, which will serve as a baseline scheme throughout the remainder of this paper.

Example 1 (Uncoded Caching). For a memory size of $M F$ bits, one possible strategy is for each user to cache the same $M / N$ fraction of each file in the placement phase. In the delivery phase, the server simply transmits the remaining $1-M / N$ fraction of any requested file over the shared link. Clearly, each user can recover its requested file from the content of its local cache and the signal sent over the shared link. In the worst case the users request different files - the delivery rate for this caching scheme is thus

$$
R_{U}(M) \triangleq K \cdot(1-M / N) \cdot \min \{1, N / K\}
$$

We refer to this caching strategy as uncoded caching, since both content placement and delivery are uncoded.

The first factor $K$ in (1) is the rate without caching. The second factor in (1) is $1-M / N$. We call this the local caching gain, since it arises from having a fraction $M / N$ of each file available locally at the user. If $N<K$, the system enjoys an additional gain, reflected in the third factor in (1). In this case, some users will necessarily request the same file, resulting in a natural multicasting gain of $N / K$.

\section{B. Formal Problem Statement}

We now provide the formal definition of the information-theoretic caching problem. Let $\left(W_{n}\right)_{n=1}^{N}$ be $N$ independent random variables each uniformly distributed over

$$
\left[2^{F}\right] \triangleq\left\{1,2, \ldots, 2^{F}\right\}
$$

for some $F \in \mathbb{N}$. Each $W_{n}$ represents a file of size $F$ bits. A $(M, R)$ caching scheme consists of $K$ caching functions, $N^{K}$ encoding functions, and $K N^{K}$ decoding functions.

The $K$ caching functions

$$
\phi_{k}:\left[2^{F}\right]^{N} \rightarrow\left[2^{\lfloor F M\rfloor}\right]
$$

map the files $W_{1}, \ldots, W_{N}$ into the cache content

$$
Z_{k} \triangleq \phi_{k}\left(W_{1}, \ldots W_{N}\right)
$$

for each user $k \in[K]$ during the placement phase. The $N^{K}$ encoding functions

$$
\psi_{\left(d_{1}, \ldots, d_{K}\right)}:\left[2^{F}\right]^{N} \rightarrow\left[2^{\lfloor F R\rfloor}\right]
$$

map the files $W_{1}, \ldots, W_{N}$ to the input

$$
X_{\left(d_{1}, \ldots, d_{K}\right)} \triangleq \psi_{\left(d_{1}, \ldots, d_{K}\right)}\left(W_{1}, \ldots, W_{N}\right)
$$

of the shared link responding to the requests $\left(d_{1}, \ldots, d_{K}\right) \in[N]^{K}$ during the delivery phase. Finally, the $K N^{K}$ decoding functions

$$
\mu_{\left(d_{1}, \ldots, d_{K}\right), k}:\left[2^{\lfloor R F\rfloor}\right] \times\left[2^{\lfloor F M\rfloor}\right] \rightarrow\left[2^{F}\right]
$$

map the signal received over the shared link $X_{\left(d_{1}, \ldots, d_{K}\right)}$ and the cache content $Z_{k}$ to the estimate

$$
\hat{W}_{\left(d_{1}, \ldots, d_{K}\right), k} \triangleq \mu_{\left(d_{1}, \ldots, d_{K}\right), k}\left(X_{\left(d_{1}, \ldots, d_{K}\right)}, Z_{k}\right)
$$


of the requested file $W_{d_{k}}$ of user $k \in[K]$. The probability of error is defined as

$$
\max _{\left(d_{1}, \ldots, d_{K}\right) \in[N]^{K}} \max _{k \in[K]} \mathbb{P}\left(\hat{W}_{\left(d_{1}, \ldots, d_{K}\right), k} \neq W_{d_{k}}\right) .
$$

Definition. The pair $(M, R)$ is achievable if for every $\varepsilon>0$ and every large enough file size $F$ there exists a $(M, R)$ caching scheme with probability of error less than $\varepsilon$. We define the memory-rate tradeoff

$$
R^{\star}(M) \triangleq \inf \{R:(M, R) \text { is achievable }\} .
$$

\section{MAin RESUltS}

The first theorem presents an achievable rate $R_{C}(M)$, yielding an upper bound on the memory-rate tradeoff $R^{\star}(M)$.

Theorem 1. For $N \in \mathbb{N}$ files and $K \in \mathbb{N}$ users each with cache of size $M \in\{0, N / K, 2 N / K, \ldots, N\}$,

$$
R^{\star}(M) \leq R_{C}(M) \triangleq K \cdot(1-M / N) \cdot \min \left\{\frac{1}{1+K M / N}, \frac{N}{K}\right\}
$$

is achievable. For general $0 \leq M \leq N$, the lower convex envelope of these points is achievable.

The rate $R_{C}(M)$ is achieved by a coded caching scheme that is described and analyzed in detail in Section $\mathrm{V}$. For ease of exposition, we first focus on the case $N \geq K$, in which

$$
R_{C}(M)=K \cdot(1-M / N) \cdot \frac{1}{1+K M / N} .
$$

The achievable rate $R_{C}(M)$ consists of three distinct factors. The first factor in $R_{C}(M)$ is $K$. This is the worst-case rate without caches at the users (i.e., $M=0$ ).

The second factor in $R_{C}(M)$ is $1-M / N$. Referring to (1) in Example 1, we see that this term, capturing the local caching gain, appears also in the rate expression of the uncoded caching scheme. Observe that this local gain is a function of the normalized local memory size $M / N$, and it is relevant whenever $M$ is on the order of $N$.

Finally, the third factor in $R_{C}(M)$ is $\frac{1}{1+K M / N}$, which we call the global caching gain. This gain is a function of the normalized global or cumulative memory size $K M / N$, and it is relevant whenever $K M$ is on the order of (or larger than) $N$. This global gain is to be interpreted as a multicasting gain available simultaneously for all possible demands. Note that, since the number of users is smaller than the number of files, in the worst case all users request different files. Hence, there are no natural multicasting opportunities. The scheme proposed in Theorem 1 carefully designs the content placement in order to create coded multicasting opportunities in the delivery phase even among users that request different files. Since the placement phase is performed without knowledge of the actual demands, care must be taken to ensure that the same multicasting opportunities are created simultaneously for every possible set of requests in the delivery phase.

We point out that the uncoded caching scheme introduced in Example 1 achieves only the local caching gain, whereas the coded caching scheme proposed in Theorem 1 achieves both the local as well as the global caching gains. The following two examples compare these two gains.

Example $2(\Theta(K)$ Improvement in Rate). Consider a system with the same number of users as files, i.e., $N=K$. Assume each user has enough cache memory for half of the files so that $M=N / 2$. Then the local caching gain is $1 / 2$ and the global caching gain is $1 /(1+K / 2)$. By (1), uncoded caching achieves a rate of $K / 2$. On the other hand, by Theorem 1, coded caching achieves a rate of $(K / 2) /(1+K / 2)<1$ : a reduction by more than a factor $K / 2$ in rate compared to the uncoded scheme. We refer the reader to Fig. 2 in Section \ for a visualization of the effect of this improvement.

Example $3(\Theta(K)$ Improvement in Slope). In this example, we compare the performance of the coded and uncoded caching schemes for small values of the cache size $M$. We consider again the case $N=K$. 
From (1), the rate of uncoded caching has a slope of -1 around $M=0$. On the other hand, by Theorem 1 , the rate of coded caching has a slope less than $-K / 2$ around $M=0.1$ Therefore, the coded caching scheme reduces the rate over the shared link at least $K / 2$ times faster as a function of cache size than the uncoded caching scheme. Comparing the rates of the uncoded and coded schemes in Fig. 2 in Section II for small values of $M$ illustrates the effect of this improvement.

Consider next the case $N<K$, in which the third factor in $R_{C}(M)$ is the minimum of $\frac{1}{1+K M / N}$ and $N / K$. The first term in this minimum is the coded multicasting gain created by careful content placement as discussed for the case $N \geq K$. However, for a scenario with fewer files than users, there exists already a natural multicasting opportunity: by multicasting all $N$ files to the $K$ users, we can achieve a gain of $N / K$. This is the second term in the minimum above. The scheme in Theorem 1 achieves the better of these two gains. We point out that for $M \geq 1-N / K$ this minimum is achieved by the first of the two gains. In other words, the natural multicasting gain is relevant only when the memory size is very small.

Having established an upper bound on the memory-rate tradeoff $R^{\star}(M)$, we proceed with a lower bound on it.

Theorem 2. For $N \in \mathbb{N}$ files and $K \in \mathbb{N}$ users each with cache of size $0 \leq M \leq N$,

$$
R^{\star}(M) \geq \max _{s \in\{1, \ldots, \min \{N, K\}\}}\left(s-\frac{s}{\lfloor N / s\rfloor} M\right) .
$$

The proof of Theorem 2, presented in Section VI, is based on a cut-set bound argument. Tighter lower bounds on $R^{\star}(M)$ can be derived using stronger arguments than the cut-set bound (see the discussion in Example 4 in Section IV]. However, the cut-set bound alone is sufficient for a constantfactor approximation of the memory-rate tradeoff $R^{\star}(M)$, as the next theorem shows by comparing the achievable rate $R_{C}(M)$ in Theorem 1 with the lower bound in Theorem 2 ,

Theorem 3. For $N \in \mathbb{N}$ files and $K \in \mathbb{N}$ users each with cache of size $0 \leq M \leq N$,

$$
1 \leq \frac{R_{C}(M)}{R^{\star}(M)} \leq 12
$$

with the achievable rate $R_{C}(M)$ of coded caching as defined in Theorem 1$]$

The proof of Theorem 3 is presented in Section VII. The bound $R_{C}(M) / R^{\star}(M) \leq 12$ on the approximation ratio of the proposed caching scheme is somewhat loose due to the analytical bounding techniques that were used. Numerical simulations suggest that

$$
\frac{R_{C}(M)}{R^{\star}(M)} \leq 5
$$

for all $N, K$, and $0 \leq M \leq N$.

Theorem 3 shows that the rate $R_{C}(M)$ of the proposed coded caching scheme in Theorem 1 is close to the information-theoretic optimum $R^{\star}(M)$ for all values of the system parameters. More precisely, it shows that no scheme can improve upon the rate $R_{C}(M)$ of the proposed scheme by more than a factor 12. This also suggests that the local and global caching gains identified in this paper are fundamental: there are no other significant caching gains (i.e., scaling with the problem parameters) beyond these two.

\section{EXAMPLES}

Example 4. Consider the case $N=K=2$, so that there are two files, say $W_{1}=A, W_{2}=B$, and two users each with cache memory of size $M$. The upper and lower bounds in Theorems 1 and 2 on the memory-rate tradeoff $R^{\star}(M)$ are depicted in Fig. 3. To illustrate the proof techniques, we now show how these two bounds are derived for this simple setting.

\footnotetext{
${ }^{1}$ This follows by calculating the slope of the straight line connecting the two consecutive corner points of $R_{C}(M)$ at $M=0$ and $M=1$.
} 


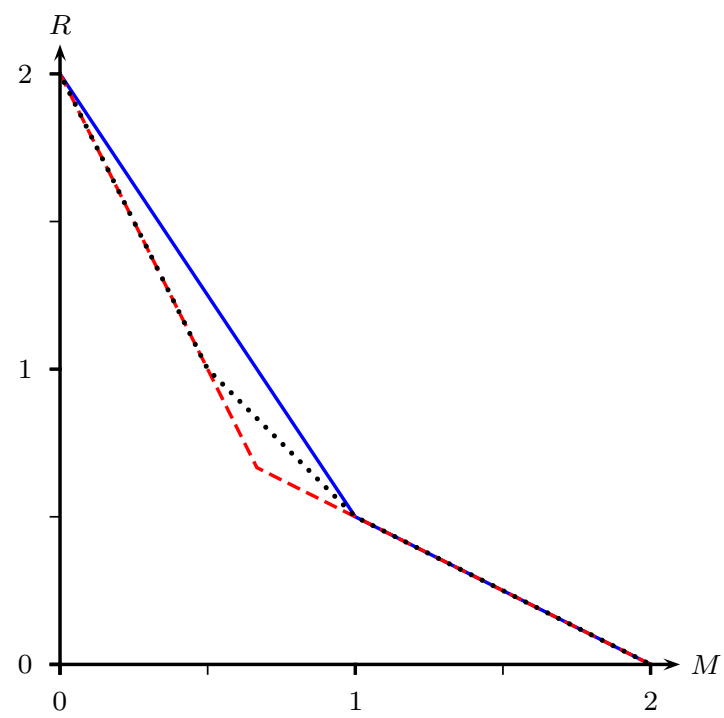

Fig. 3. Memory-rate tradeoff for $N=2$ files $K=2$ users. The achievable rate $R_{C}(M)$ of the coded caching scheme from Theorem 1 is indicated by the solid blue curve. The lower bound on $R^{\star}(M)$ from Theorem 2 is indicated by the dashed red curve. For the $N=K=2$ case, $R^{\star}(M)$ can be found exactly and is indicated by the dotted black curve.

We start with the upper bound in Theorem 1, focusing on the corner points of the achievable region. First, let us consider the two extreme cases $M=0$ and $M=N$. If $M=0$, the server can always transmit both files $A$ and $B$ over the shared link. Since this satisfies every possible request, the $(M, R)$ pair $(0,2)$ is achievable. If $M=2$, each user can cache both files $A$ and $B$ in the placement phase. Therefore, no communication is needed in the delivery phase and the $(M, R)$ pair $(2,0)$ is achievable.
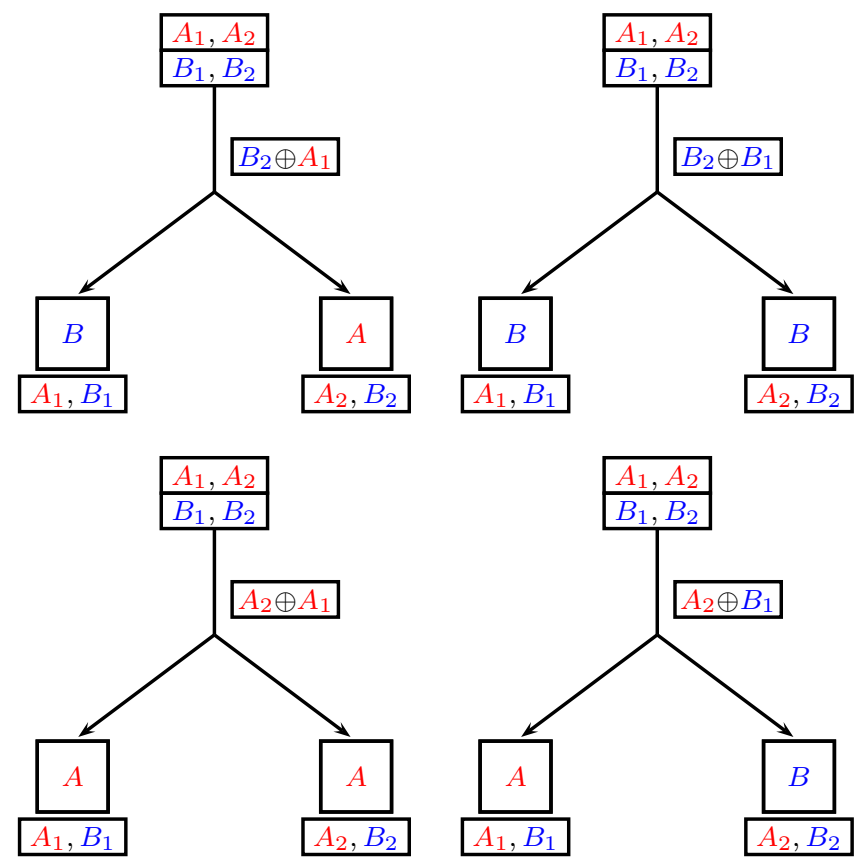

Fig. 4. Caching strategy for $N=2$ files and $K=2$ users with cache size $M=1$ with all four possible user requests. Each file is split into two subfiles of size $1 / 2$, i.e., $A=\left(A_{1}, A_{2}\right)$ and $B=\left(B_{1}, B_{2}\right)$. The scheme achieves rate $R=1 / 2$. Observe that, while the transmission from the server changes as a function of the user requests, the cache contents do not.

Consider then the more interesting corner point at $M=1$. The caching scheme achieving the upper bound in Theorem 1 is as follows (see Fig. 4). We split both files $A$ and $B$ into two subfiles of equal size, 
i.e., $A=\left(A_{1}, A_{2}\right)$ and $B=\left(B_{1}, B_{2}\right)$. In the placement phase, we set $Z_{1}=\left(A_{1}, B_{1}\right)$ and $Z_{2}=\left(A_{2}, B_{2}\right)$. In words, each user caches one exclusive part of each file. For the delivery phase, assume for example that user one requests file $A$ and user two requests file $B$. Given that user one already has subfile $A_{1}$ of $A$, it only needs to obtain the missing subfile $A_{2}$, which is cached in the second user's memory $Z_{2}$. Similarly, user two only needs to obtain the missing subfile $B_{1}$, which is cached in the first user's memory $Z_{1}$. In other words, each user has one part of the file that the other user needs.

The server can in this case simply transmit $A_{2} \oplus B_{1}$, where $\oplus$ denotes bitwise XOR. Since user one already has $B_{1}$, it can recover $A_{2}$ from $A_{2} \oplus B_{1}$. Similarly, since user two already has $A_{2}$, it can recover $B_{1}$ from $A_{2} \oplus B_{1}$. Thus, the signal $A_{2} \oplus B_{1}$ received over the shared link helps both users to effectively exchange the missing subfiles available in the cache of the other user.

The signals sent over the shared link for all other requests are depicted in Fig. 4, One can see that in all cases the signal is constructed using the same logic of exchanging the missing subfiles. This proves the achievability of the $(M, R)$ pair $(1,1 / 2)$.

It is worth pointing out that in each case the server sends a single coded multicast transmission to satisfy two (possibly different) user requests. Moreover, these coded multicasting opportunities are available simultaneously for all four possible user requests. This availability of simultaneous multicasting opportunities, enabled by careful content placement, is critical, since the placement phase has to be performed without knowledge of the actual demands in the delivery phase.

So far, we have shown that the $(M, R)$ pairs at corner points $(0,2),(1,1 / 2)$, and $(2,0)$ are achievable. On the other hand, by dividing the cache memories and the transmitted signal proportionally, it is easy to see that if any two points $\left(M_{1}, R_{1}\right)$ and $\left(M_{2}, R_{2}\right)$ are achievable, then the line connecting them is also achievable. Inspired by the term time sharing in network information theory, we refer to this as memory sharing.

Memory sharing between the corner points $(0,2),(1,1 / 2)$, and $(2,0)$ establishes the achievability of the solid blue curve in Fig. 3, which coincides with the upper bound stated in Theorem 1.

We continue by analyzing the lower bound on $R^{\star}(M)$ in Theorem 2 . The proof relies on the cut-set bound. We consider two cuts. The first cut separates $\left(X_{(1,2)}, Z_{1}, Z_{2}\right)$ from the two users. Assume $(M, R)$ is an achievable memory-rate pair. Then this cut has capacity at most $R F+2 M F$, since $X_{(1,2)}$ is at most $R F$ bits by definition of achievability, and since $Z_{1}, Z_{2}$ contain each $M F$ bits. On the other hand, since the first user can recover $A$ from $\left(X_{(1,2)}, Z_{1}\right)$ and the second user can recover $B$ from $\left(X_{(1,2)}, Z_{2}\right)$, the number of bits that need to be transmitted over this cut is at least $2 F$. Hence,

$$
R F+2 M F \geq 2 F
$$

so that

$$
R \geq 2-2 M .
$$

As this holds for all achievable memory-rate pairs $(M, R)$, we conclude that

$$
R^{\star}(M) \geq 2-2 M \text {. }
$$

The second cut separates $\left(X_{(1,2)}, X_{(2,1)}, Z_{1}\right)$ from the first user. Note that this user can recover $A$ and $B$ from $\left(X_{(1,2)}, Z_{1}\right)$ and $\left(X_{(2,1)}, Z_{1}\right)$, respectively. Hence, this cut yields

$$
2 R F+M F \geq 2 F
$$

for any achievable memory-rate pair $(M, R)$, implying that

$$
R^{\star}(M) \geq 1-M / 2 .
$$

Together, this yields the dashed red curve in Fig. 3, which coincides with the lower bound stated in Theorem 2 . 
For the case $N=K=2$, the memory-rate tradeoff can in fact be found exactly and is indicated by the dotted black curve in Fig. 3. This is argued by showing that the pair $(M, R)=(1 / 2,1)$ is also achievable, and by deriving the additional non cut-set bound

$$
R^{\star}(M) \geq 3 / 2-M .
$$

This shows that, while the bounds in Theorems 1 and 2 are sufficient to characterize the memory-rate tradeoff $R^{\star}(M)$ to within a constant multiplicative gap, neither the achievable region nor the lower bound are tight in general. The details of this derivation are reported in the Appendix.

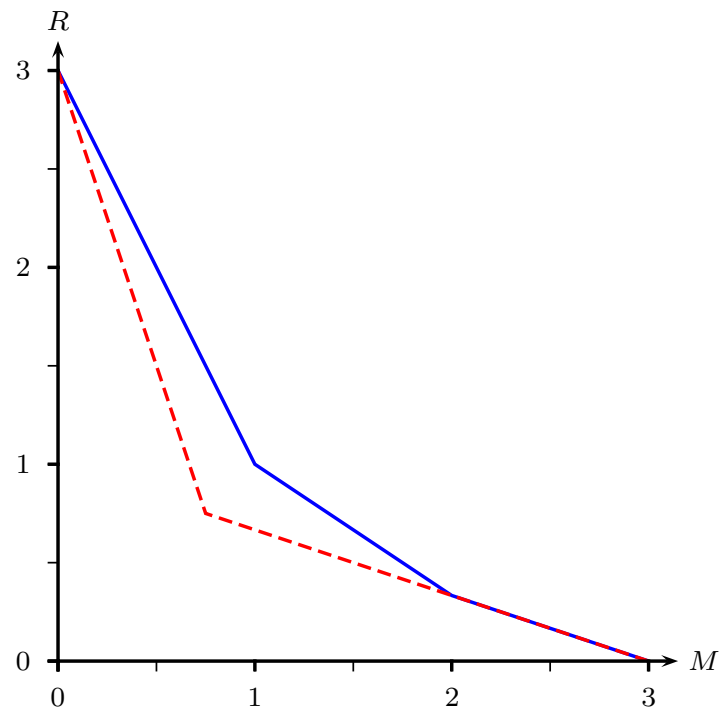

Fig. 5. Memory-rate tradeoff for $N=3$ files and $K=3$ users. The achievable rate $R_{C}(M)$ of the coded caching scheme from Theorem 1 is indicated by the solid blue curve. The lower bound on $R^{\star}(M)$ from Theorem 2 is indicated by the dashed red curve.

Example 5. In this example, we assume that $N=K=3$ so that there are three users and three files, say $W_{1}=A, W_{2}=B$, and $W_{3}=C$. Again, it is trivial to see that the $(M, R)$ pairs $(0,3)$ and $(3,0)$ are achievable. We focus on two nontrivial corner points at $M=1$ and $M=2$.

Consider first caches of size $M=1$. We split each file into three subfiles of equal size, i.e., $A=$ ( $\left.A_{1}, A_{2}, A_{3}\right), B=\left(B_{1}, B_{2}, B_{3}\right)$, and $C=\left(C_{1}, C_{2}, C_{3}\right)$. In the placement phase, the cache content of user $k$ is selected as $Z_{k}=\left(A_{k}, B_{k}, C_{k}\right)$. A more formal way to describe this content placement, which is a bit exaggerated for this simple setting but will be useful for the general setting below, is as follows. Let $\mathcal{T}$ be a subset of one element of $\{1,2,3\}$. Then subfiles $A_{\mathcal{T}}, B_{\mathcal{T}}, C_{\mathcal{T}}$ are placed into the cache of user $k$ if $k \in \mathcal{T}$. For example, $A_{1}$ is cached at user one since in this case $\mathcal{T}=\{1\}$.

For the delivery phase, let us consider as an example that user one requests file $A$, user two requests file $B$, and user three requests file $C$. Then the missing subfiles are $A_{2}$ and $A_{3}$ for user one, $B_{1}$ and $B_{3}$ for user two, and $C_{1}$ and $C_{2}$ for user three. Given the cache contents, users one and two aim to exchange $A_{2}$ and $B_{1}$, users one and three aim to exchange $A_{3}$ and $C_{1}$, and users two and three aim to exchange $B_{3}$ and $C_{2}$. The signal $\left(A_{2} \oplus B_{1}, A_{3} \oplus C_{1}, B_{3} \oplus C_{2}\right)$ enables all of these three exchanges. All other requests can be satisfied in a similar manner. Since each subfile has rate $1 / 3$, the proposed scheme achieves a rate of 1 , and therefore $(M, R)$ pair $(1,1)$ is achievable.

Observe that, through careful content placement, we have again created coded multicasting opportunities for any two users even with different demands. Moreover, these coded multicasting opportunities are available simultaneously for all 27 possible triples of user requests.

Consider next caches of size $M=2$. We again split each file into three subfiles of equal size. However, it will be convenient to label these subfiles differently, namely $A=\left(A_{12}, A_{13}, A_{23}\right), B=\left(B_{12}, B_{13}, B_{23}\right)$, 
and $C=\left(C_{12}, C_{13}, C_{23}\right)$. In the placement phase, the caching strategy is

$$
\begin{aligned}
& Z_{1}=\left(A_{12}, A_{13}, B_{12}, B_{13}, C_{12}, C_{13}\right), \\
& Z_{2}=\left(A_{12}, A_{23}, B_{12}, B_{23}, C_{12}, C_{23}\right), \\
& Z_{3}=\left(A_{13}, A_{23}, B_{13}, B_{23}, C_{13}, C_{23}\right) .
\end{aligned}
$$

This content placement can be understood as follows. Let $\mathcal{T}$ be a subset of two elements of $\{1,2,3\}$. Then subfiles $A_{\mathcal{T}}, B_{\mathcal{T}}, C_{\mathcal{T}}$ are placed into the cache of user $k$ if $k \in \mathcal{T}$. For example, $A_{13}$ is cached at users one and three since in this case $\mathcal{T}=\{1,3\}$.

For the delivery phase, let us again assume as an example that user one requests file $A$, user two requests file $B$, and user three requests file $C$. In this case, user one misses subfile $A_{23}$, which is available at both users two and three. User two misses subfile $B_{13}$, which is available at both users one and three. And user three misses subfile $C_{12}$, which is available at both users one and two. In other words, the three users would like to exchange the subfiles $A_{23}, B_{13}, C_{12}$. This exchange can be enabled by transmitting the signal $A_{23} \oplus B_{13} \oplus C_{12}$ over the shared link. Given its cache content, each user can then recover the missing subfile. All other requests can be satisfied in a similar manner. The rate of transmission in the delivery phase is $1 / 3$, and therefore $(M, R)$ pair $(2,1 / 3)$ is achievable. This approach again creates simultaneous coded multicasting opportunities, but this time for all three users together.

The arguments so far show that the solid blue curve in Fig. 5 is achievable. This coincides with the upper bound in Theorem 1

For the lower bound on $R^{\star}(M)$, we use two cut-set bounds. The first cut separates $\left(X_{(1,2,3)}, Z_{1}, Z_{2}, Z_{3}\right)$ from the three users. Note that the users can recover $A, B$, and $C$, from $\left(X_{(1,2,3)}, Z_{1}\right),\left(X_{(1,2,3)}, Z_{2}\right)$, and $\left(X_{(1,2,3)}, Z_{3}\right)$, respectively. For any achievable $(M, R)$ pair, the capacity of this cut is at most $R F+3 M F$, and the number of bits that need to be transmitted over it is at least $3 F$. Hence,

$$
R F+3 M F \geq 3 F
$$

which implies that

$$
R^{\star}(M) \geq 3-3 M
$$

The second cut separates $\left(X_{(1,2,3)}, X_{(2,3,1)}, X_{(3,1,2)}, Z_{1}\right)$ from the first user. Note that this user can recover $A, B$, and $C$ from $\left(X_{(1,2,3)}, Z_{1}\right),\left(X_{(2,3,1)}, Z_{1}\right)$, and $\left(X_{(3,1,2)}, Z_{1}\right)$, respectively. For any achievable $(M, R)$ pair, the cut capacity is at most $3 R F+M F$, and the number of bits that need to be transmitted over it is at least $3 F$. Hence,

$$
3 R F+M F \geq 3 F
$$

which implies that

$$
R^{\star}(M) \geq 1-M / 3 \text {. }
$$

Together, these two cut-set bounds result in the dashed red curve in Fig. 5. This coincides with the lower bound in Theorem 2 ,

\section{Coded Caching Scheme (Proof of Theorem 1)}

We now present the general achievable scheme. We first describe the algorithm in words, focusing on the corner points of $R_{C}(M)$. Consider cache size $M \in\{0, N / K, 2 N / K, \ldots, N\}$, and set

$$
t \triangleq M K / N \text {. }
$$

Observe that $t$ is an integer between 0 and $K$.

If $M=0$, then in the delivery phase the server can simply transmit the union of all requested files over the shared link, resulting in $F \min \{N, K\}$ bits being sent. Hence

$$
R^{\star}(0) \leq \min \{N, K\} .
$$


If $M=N$, then all files in the database can be cached at every user in the placement phase. Hence

$$
R^{\star}(N)=0 .
$$

Assume in the following that $M$ is strictly larger than zero and strictly less than $N$, so that $t \in$ $\{1,2, \ldots, K-1\}$.

In the placement phase, each file is split into $\left(\begin{array}{c}K \\ t\end{array}\right)$ nonoverlapping subfiles of equal size. It will be convenient to label the subfiles of file $W_{n}$ as

$$
W_{n}=\left(W_{n, \mathcal{T}}: \mathcal{T} \subset[K],|\mathcal{T}|=t\right),
$$

where we recall the notation

$$
[K] \triangleq\{1, \ldots, K\} .
$$

For each $n \in[N]$, subfile $W_{n, \mathcal{T}}$ is placed in the cache of user $k$ if $k \in \mathcal{T}$. Thus, each user caches a total of $N\left(\begin{array}{c}K-1 \\ t-1\end{array}\right)$ subfiles. Since each of these subfiles has size $F /\left(\begin{array}{c}K \\ t\end{array}\right)$, this requires

$$
N\left(\begin{array}{c}
K-1 \\
t-1
\end{array}\right) \frac{F}{\left(\begin{array}{c}
K \\
t
\end{array}\right)}=F \frac{N t}{K}=F M
$$

bits of cache memory at each user, satisfying the memory constraint.

Example 6. Let $N=K=3$ and $M=2$. Then $t=2$ and the content placement is

$$
\begin{aligned}
& Z_{1}=\left(W_{n,\{1,2\}}, W_{n,\{1,3\}}\right)_{n=1}^{3}, \\
& Z_{2}=\left(W_{n,\{1,2\}}, W_{n,\{2,3\}}\right)_{n=1}^{3}, \\
& Z_{3}=\left(W_{n,\{1,3\}}, W_{n,\{2,3\}}\right)_{n=1}^{3},
\end{aligned}
$$

as we have already seen in Example 5 in Section IV.

We next describe the delivery phase. Consider the request vector $\left(d_{1}, \ldots, d_{K}\right) \in[N]^{K}$, i.e., user $k$ requests file $W_{d_{k}}$. We focus on a subset $\mathcal{S} \subset[K]$ of $|\mathcal{S}|=t+1$ users. Observe that every $t$ users in $\mathcal{S}$ share a subfile in their caches that is needed at the remaining user in $\mathcal{S}$. More precisely, fix a user $s \in \mathcal{S}$, and note that $|\mathcal{S} \backslash\{s\}|=t$. The subfile $W_{d_{s}, \mathcal{S} \backslash\{s\}}$ is requested by user $s$ since it is a subfile of $W_{d_{s}}$. At the same time, it is missing at user $s$ since $s \notin \mathcal{S} \backslash\{s\}$. Finally, it is present in the cache of any user $k \in \mathcal{S} \backslash\{s\}$.

For each such subset $\mathcal{S} \subset[K]$ of cardinality $|\mathcal{S}|=t+1$, the server transmits

$$
\oplus_{s \in \mathcal{S}} W_{d_{s}, S \backslash\{s\}},
$$

where $\oplus$ denotes bitwise XOR. Each of these sums results in $F /\left(\begin{array}{c}K \\ t\end{array}\right)$ bits being sent over the shared link. Since the number of subsets $\mathcal{S}$ is $\left(\begin{array}{c}K \\ t+1\end{array}\right)$, the total number of bits sent over the shared link is

$$
\begin{aligned}
F R & =\left(\begin{array}{c}
K \\
t+1
\end{array}\right) \frac{F}{\left(\begin{array}{c}
K \\
t
\end{array}\right)} \\
& =F \frac{K-t}{t+1} \\
& =F \frac{K(1-M / N)}{1+K M / N},
\end{aligned}
$$

where we have used that $t=M K / N$.

Example 7. Let $N=K=3$ and $M=1$. Then $t=1$ and the content placement is $Z_{k}=\left(W_{n,\{k\}}\right)_{n=1}^{3}$. For request $\left(d_{1}, d_{2}, d_{3}\right)=(1,2,3)$, the signal transmitted in the delivery phase is

$$
X_{(1,2,3)}=\left(W_{1,\{2\}} \oplus W_{2,\{1\}}, W_{1,\{3\}} \oplus W_{3,\{1\}}, W_{2,\{3\}} \oplus W_{3,\{2\}}\right),
$$


as we have already seen in Example 5 in Section IV] Here, the three sums correspond to $\mathcal{S}=\{1,2\}$, $\mathcal{S}=\{3,1\}$, and $\mathcal{S}=\{3,2\}$, respectively.

We now argue that each user can successfully recover its requested message. Consider again a subset $\mathcal{S} \subset[K]$ with $|\mathcal{S}|=t+1$. Since each user $k \in \mathcal{S}$ already has access to the subfiles $W_{d_{s}, S \backslash\{s\}}$ for all $s \in \mathcal{S} \backslash\{k\}$, it can solve for $W_{d_{k}, S \backslash\{k\}}$ from the signal

$$
\oplus_{s \in \mathcal{S}} W_{d_{s}, S \backslash\{s\}}
$$

sent over the shared link. Since this is true for every such subset $\mathcal{S}$, each receiver $k$ is able to recover all subfiles of the form

$$
\left\{W_{d_{k}, \mathcal{T}}: \mathcal{T} \subset[K] \backslash\{k\},|\mathcal{T}|=t\right\}
$$

of the requested file $W_{d_{k}}$. The remaining subfiles are of the form $W_{d_{k}, \mathcal{T}}$ for $\mathcal{T}$ such that $k \in \mathcal{T}$. But these subfiles are already available in the cache of user $k$. Hence each user $k$ can recover all subfiles of its requested file $W_{d_{k}}$.

This shows that, for $M \in\{N / K, 2 N / K, \ldots,(K-1) N / K\}$,

$$
R^{\star}(M) \leq K \cdot(1-M / N) \cdot \frac{1}{1+K M / N} .
$$

As was pointed out earlier (see Example 4 in Section IV], the points on the line connecting any two achievable points are also achievable. Finally, taking the minimum between the rate derived here and the rate

$$
K \cdot(1-M / N) \cdot \min \{1, N / K\}
$$

achieved by the conventional uncoded scheme described in Example 1 in Section III-A proves Theorem 1

For completeness, in Algorithm 1, we formally describe the placement and the delivery procedures of the coded caching scheme for $N$ files, $K$ users. We focus on the corner points of $R_{C}(M)$, which occur at cache size $M$ such that $M K / N$ is a positive integer less than $K$.

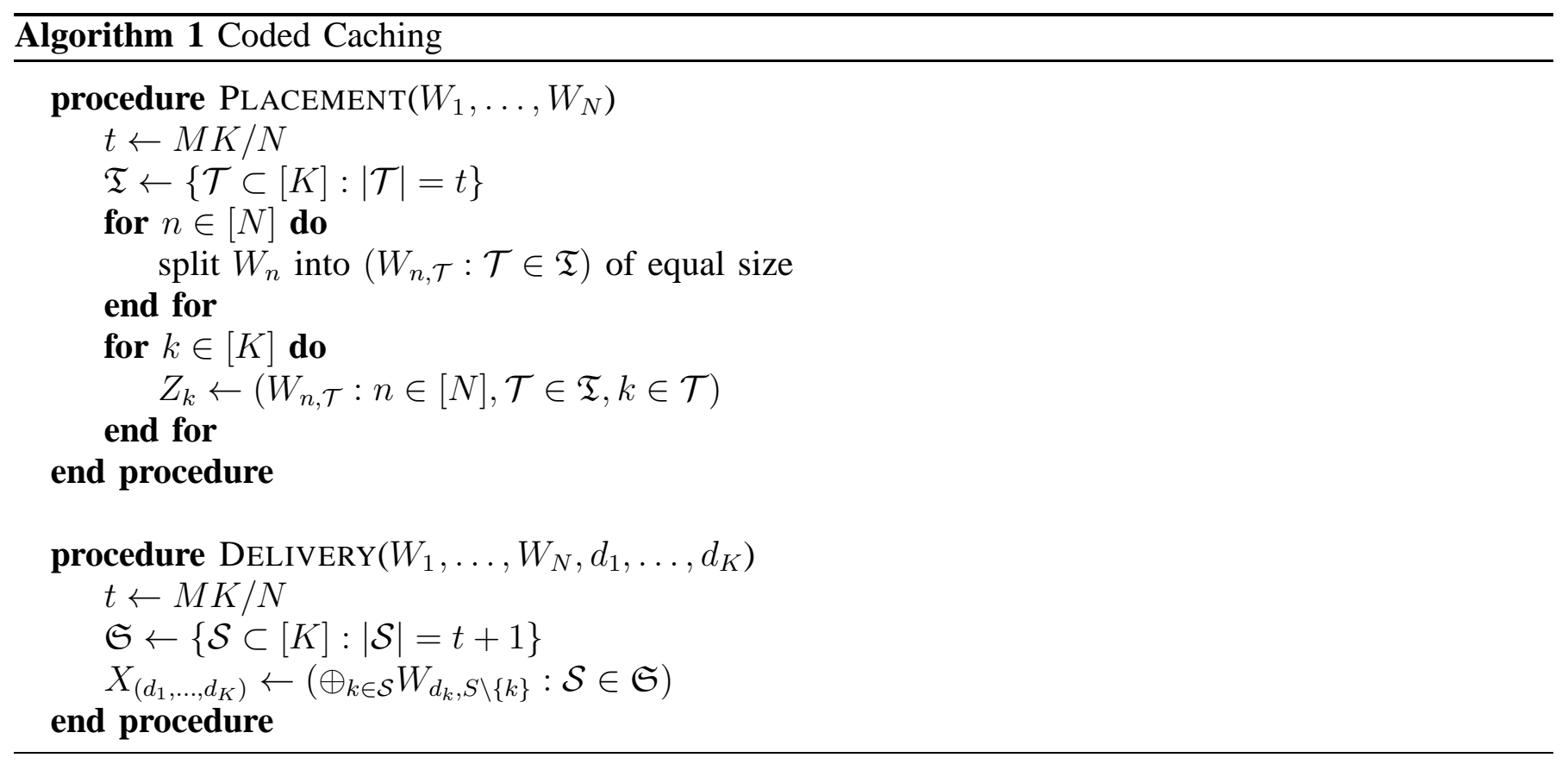

Observe that the placement phase of the algorithm is designed such that, in the delivery phase, the caches enable coded multicasting between $M K / N+1$ users with different demands. These coded multicasting 
opportunities are available simultaneously for all $N^{K}$ possible user demands. This is again critical, since the placement phase has to be designed without knowledge of the actual demands in the delivery phase.

We also point out that while the problem setting allows for vanishing probability of error as $F \rightarrow \infty$, the achievable scheme presented here actually has zero error probability for finite $F$.

\section{LOWER BOUND ON $R^{\star}(M)$ (PROOF OF THEOREM 2)}

Let $s \in\{1, \ldots, \min \{N, K\}\}$ and consider the first $s$ caches $Z_{1}, \ldots, Z_{s}$. There exists a user demand and a corresponding input to the shared link, say $X_{1}$, such that $X_{1}$ and $Z_{1}, \ldots, Z_{s}$ determine the files $W_{1}, \ldots, W_{s}$. Similarly, there exists an input to the shared link, say $X_{2}$, such that $X_{2}$ and $Z_{1}, \ldots, Z_{s}$ determine the files $W_{s+1}, \ldots, W_{2 s}$. Continue in the same manner selecting appropriate $X_{3}, \ldots, X_{\lfloor N / s\rfloor}$, see Fig. 6. We then have that $X_{1}, \ldots, X_{\lfloor N / s\rfloor}$ and $Z_{1}, \ldots, Z_{s}$ determine $W_{1}, \ldots, W_{s\lfloor N / s\rfloor}$.

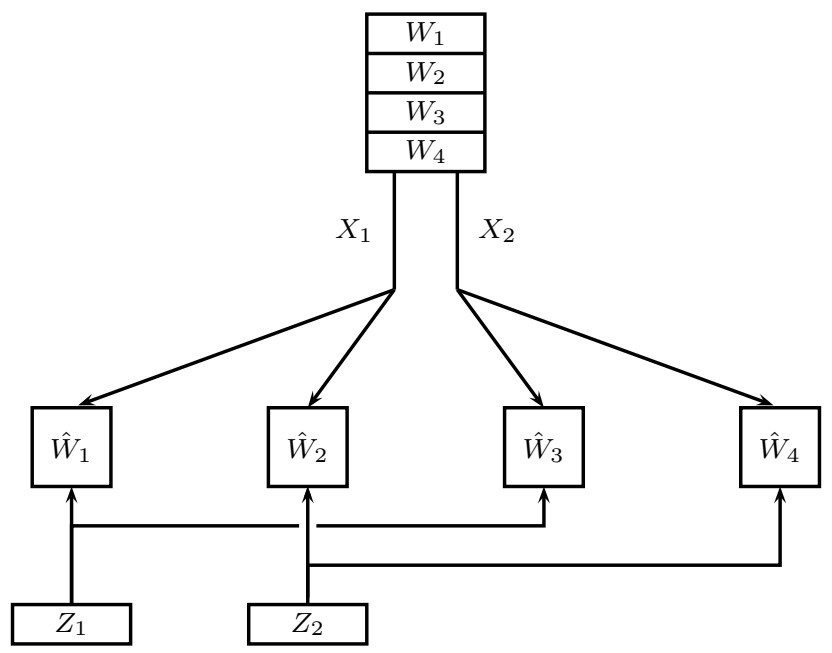

Fig. 6. Cut corresponding to parameter $s=2$ in the proof of the converse. In the figure, $N=K=4$, and $X_{1}=X_{(1,2,3,4)}, X_{2}=X_{(3,4,2,1)}$.

Consider then the cut separating $X_{1}, \ldots, X_{\lfloor N / s\rfloor}$ and $Z_{1}, \ldots, Z_{s}$ from the corresponding users as indicated in Fig. 6. By the cut-set bound [10, Theorem 14.10.1],

$$
\lfloor N / s\rfloor R^{\star}(M)+s M \geq s\lfloor N / s\rfloor .
$$

Solving for $R^{\star}$ and optimizing over all possible choices of $s$, we obtain

$$
R^{\star}(M) \geq \max _{s \in\{1, \ldots, \min \{N, K\}\}}\left(s-\frac{s}{\lfloor N / s\rfloor} M\right),
$$

proving the theorem.

\section{Approximation of $R^{\star}(M)$ (Proof of Theorem 3)}

We now compare the upper bound on $R^{\star}(M)$ in Theorem 2 to the rate $R_{C}(M)$ achieved by the proposed scheme given by the lower convex envelope of the points

$$
R_{C}(M)=\min \left\{\frac{K(1-M / N)}{1+K M / N}, N-M\right\}
$$

for $M$ a multiple of $N / K$ as described in Theorem 11. The proof is based on the following observation. The function $R_{C}(M)$ has three distinct regimes as depicted in Fig. (7) The first regime is for $M$ between 0 and approximately $\max \{2, N / K\}$. In this regime, $R_{C}(M)$ is close to linear. The second regime is for $M$ between approximately $\max \{2, N / K\}$ and $N / 2$, in which $R_{C}(M)$ is nonlinear and behaves essentially like $N / M$. The third regime is for $M$ between approximately $N / 2$ and $N$, in which $R_{C}(M)$ is again close 


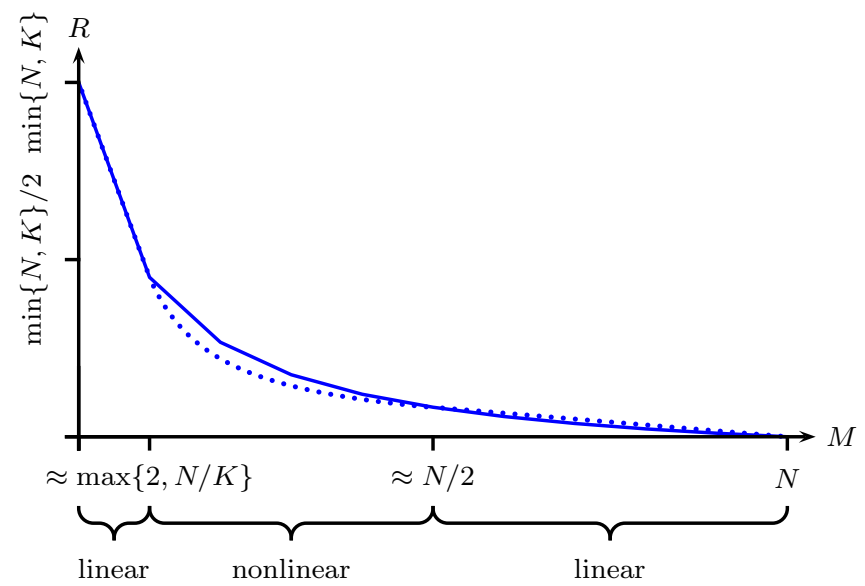

Fig. 7. Achievable rate $R_{C}(M)$ (solid curve) as defined in Theorem 1 for $N=20$ files and $K=10$ users. The function $R_{C}(M)$ has three distinct regimes, which are approximately $0 \leq M \leq \max \{2, N / K\}, \max \{2, N / K\}<M \leq N / 2$, and $N / 2<M \leq N$. In the first and third regimes, $R_{C}(M)$ is essentially linear; in the second regime, $R_{C}(M)$ is nonlinear (as indicated by the dotted curve).

to linear. We bound the ratio $R^{\star}(M) / R_{C}(M)$ separately in each of these regimes-though, to optimize the constants, we will choose slightly different definitions of the boundaries for the three regions.

It will be convenient to consider the two cases $\min \{N, K\} \leq 12$ and $\min \{N, K\} \geq 13$ separately. We have

$$
R_{C}(M) \leq \min \{N, K\}(1-M / N)
$$

by Theorem 1, On the other hand, setting $s=1$ in Theorem 2 yields

$$
R^{\star}(M) \geq 1-M / N \text {. }
$$

Hence,

$$
\frac{R_{C}(M)}{R^{\star}(M)} \leq \min \{N, K\} \leq 12
$$

for $\min \{N, K\} \leq 12$.

Assume in the following that $\min \{N, K\} \geq 13$. We consider the three cases $0 \leq M \leq 1.1 \max \{1, N / K\}$, $1.1 \max \{1, N / K\}<M \leq 0.092 N$, and $0.092 N<M \leq N$ separately. Assume first that $0 \leq M \leq$ $1.1 \max \{1, N / K\}$. We have

$$
R_{C}(M) \leq R_{C}(0) \leq \min \{N, K\}
$$

by Theorem 1, By Theorem 2, and using that $\lfloor N / s\rfloor \geq N / s-1$,

$$
R^{\star}(M) \geq s-\frac{s^{2}}{1-s / N} \frac{M}{N} .
$$

Setting $s=\lfloor 0.275 \min \{N, K\}\rfloor \in\{1, \ldots, \min \{N, K\}\}$, we obtain for $M \leq 1.1 \max \{1, N / K\}$

$$
\begin{aligned}
R^{\star}(M) & \geq R^{\star}(1.1 \max \{1, N / K\}) \\
& \geq\lfloor 0.275 \min \{N, K\}\rfloor-\frac{(\lfloor 0.275 \min \{N, K\}\rfloor)^{2}}{1-\lfloor 0.275 \min \{N, K\}\rfloor / N} \frac{1.1 \max \{1, N / K\}}{N} \\
& \geq \min \{N, K\}\left(0.275-\frac{1}{\min \{N, K\}}-\frac{1.1 \cdot 0.275^{2}}{1-0.275 \min \{1, K / N\}}\right) \\
& \geq \min \{N, K\}\left(0.275-\frac{1}{13}-\frac{1.1 \cdot 0.275^{2}}{1-0.275}\right) \\
& \geq \frac{1}{12} \min \{N, K\},
\end{aligned}
$$


where we have used the assumption that $\min \{N, K\} \geq 13$. Combining (4) and (5) yields

$$
\frac{R_{C}(M)}{R^{\star}(M)} \leq 12
$$

for $0 \leq M \leq 1.1 \max \{1, N / K\}$ and $\min \{N, K\} \geq 13$.

Assume next that $1.1 \max \{1, N / K\}<M \leq 0.092 N$. Let $\tilde{M}$ be the largest multiple of $N / K$ less than or equal to $M$, so that

$$
0 \leq M-N / K \leq \tilde{M} \leq M .
$$

Then, by Theorem 1 ,

$$
\begin{aligned}
R_{C}(M) & \leq R_{C}(\tilde{M}) \\
& \leq \frac{K(1-\tilde{M} / N)}{1+K \tilde{M} / N} \\
& \leq \frac{K(1-M / N+1 / K)}{1+K M / N-1} \\
& \leq N / M,
\end{aligned}
$$

where we have used that $M / N \geq 1 / K$ in the last inequality. Setting $s=\lfloor 0.3 N / M\rfloor \in\{1, \ldots, \min \{N, K\}\}$ in Theorem 2, we obtain

$$
\begin{aligned}
R^{\star}(M) & \geq s-\frac{s^{2}}{N-s} M \\
& \geq \frac{0.3 N}{M}-1-\frac{0.3^{2} N^{2} / M^{2}}{N-0.3 N / M} M \\
& \geq \frac{N}{M}\left(0.3-0.092-\frac{0.3^{2}}{1-0.3 / 1.1}\right) \\
& \geq \frac{N}{12 M} .
\end{aligned}
$$

Combining (7) and (8) yields

$$
\frac{R_{C}(M)}{R^{\star}(M)} \leq 12
$$

for $1.1 \max \{1, N / K\}<M \leq 0.092 N$.

Finally, assume $0.092 N<M \leq N$. Let $\tilde{M}$ be the largest multiple of $N / K$ less than or equal to $0.092 N$, so that

$$
0 \leq 0.092 N-N / K \leq \tilde{M} \leq 0.092 N .
$$

Then, using convexity of $R_{C}(\cdot)$ and that $R_{C}(N)=0$,

$$
\begin{aligned}
R_{C}(M) & \leq \frac{R_{C}(\tilde{M})}{1-\tilde{M} / N}(1-M / N) \\
& \leq \frac{1}{1-0.092} R_{C}(\tilde{M})(1-M / N),
\end{aligned}
$$

where we have used that $\tilde{M} \leq 0.092 N$. Now, by Theorem 1,

$$
\begin{aligned}
R_{C}(\tilde{M}) & \leq \frac{1}{\tilde{M} / N+1 / K} \\
& \leq \frac{1}{0.092-1 / K+1 / K} \\
& =\frac{1}{0.092} .
\end{aligned}
$$


Hence,

$$
\begin{aligned}
R_{C}(M) & \leq \frac{1}{0.092(1-0.092)}(1-M / N) \\
& \leq 12(1-M / N)
\end{aligned}
$$

Setting $s=1$ in Theorem 2, we obtain

$$
R^{\star}(M) \geq 1-M / N
$$

Combining (10) and (11) yields

$$
\frac{R_{C}(M)}{R^{\star}(M)} \leq 12
$$

for $0.092 N<M \leq N$.

Combining (3), (6), (9), and (12) yields

$$
\frac{R_{C}(M)}{R^{\star}(M)} \leq 12
$$

for all $N, K$ and all $0 \leq M \leq N$.

\section{DISCUSSION AND DIRECTIONS FOR FUTURE WORK}

We now discuss the connection of the caching problem to the index and network coding problems and list some follow-up work as well as directions for future research.

\section{A. Connection to Index and Network Coding}

The caching problem introduced in this paper is related to the index coding problem [8], [9] (or, equivalently [11], the network coding problem [12]). We now explain this connection.

The caching problem consists of a placement phase and a delivery phase. The most important aspect of this problem is the design of the placement phase in order to facilitate the delivery phase for any possible user demands. Now, for fixed content placement and for fixed demands, the delivery phase of the caching problem induces a so-called index coding problem. However, it is important to realize that the caching problem actually consists of exponentially many parallel such index coding problems, one for each of the $N^{K}$ possible user demands. Furthermore, the index coding problem itself is computationally hard to solve even only approximately [13].

The main contribution of this paper is to design the content placement such that each of the exponentially many parallel index coding problems has simultaneously an efficient and analytical solution. This, in turn, allows to solve the caching problem within a constant factor of optimality. The proposed coded caching scheme is based on prefetching uncoded raw bits and on delivering linearly encoded messages. This contrasts with the index coding problem, where linear codes are not sufficient to operate within a bounded factor of optimality (i.e., nonlinear codes can offer unbounded gain) [14].

\section{B. Decentralized Caching}

The coded caching scheme described in this paper has a placement phase that is orchestrated by a central server. Crucially for this scheme, both the number and the identity of the users in the delivery phase are already known in the prior placement phase. This is clearly not a realistic assumption since we usually do not know in the morning which users will request content in the following evening. Moreover, if instead of the synchronized user requests here we have more realistic asynchronous requests, then users join and leave the system over a period of several hours during the delivery phase, resulting in a time-varying number of users. Finally, users may be in different networks during the two phases, e.g., a 
mobile connected to a wireless local area network (e.g. WiFi) during the placement phase and connected to a cellular network (e.g. LTE) during the delivery phase.

To deal with these issues, we need a placement phase that is decentralized. We have developed such a decentralized coded caching scheme in follow-up work [15], in which each user caches a randomly selected subset of the bits. We show that the rate of this scheme is within a constant factor of optimal universally for any number of users $K$. Using this universality property allows to address the problem of asynchronous user requests and of differing networks during the two phases.

\section{Online Caching}

The caching problem here has two distinct phases: placement and delivery. The cache is updated only during the placement phase, but not during the delivery phase. Many caching systems used in practice use online cache updates, in which a decision to update the cache is made during file delivery. One popular update rule is least-recently used (better known by its abbreviation LRU), in which the least-recently requested file is evicted from the cache [16].

It is hence of interest to develop an online version of the coded caching algorithm proposed here. We present preliminary results in this direction in [17], where we introduce a coded least-recently sent delivery and update rule. For a probabilistic model of user requests, this update rule is shown to be approximately optimal in [17]. An open question is to find schemes that have stronger competitive optimality guarantees for individual sequences of user requests as in [16].

\section{Nonuniform File Popularities}

Throughout this paper, we have adopted a worst-case definition of rate with respect to user requests. However, different pieces of content have usually different popularities (i.e., probabilities of being requested by the users). In order to capture this effect, the definition of rate needs to be changed from worst case to expected value.

We report initial results extending the coded caching approach to this setting in [18]. The optimality results there are however weaker, providing only an approximation to within a factor that is (in most cases of interest) logarithmic in the number of users. Finding better schemes for this important setting of nonuniform file popularities and more precisely quantifying the impact of the file distribution on the global gain arising from coding is hence of interest. Furthermore, the results in [18] hold only when all users have the same file popularity distribution. Analyzing the impact of different per-user file popularities is an open problem.

\section{E. More General Networks}

The discussion in this paper focuses on a basic caching network consisting of a single shared link. To be relevant in practice, the results here need to be extended to more general networks. We report extensions to tree networks with caches at the leaves as well as initial results on caches shared among several users in [15]. An interesting extension of the coded caching approach proposed in this paper to device-to-device networks without a central server is reported in [19]. Adapting coded caching to general networks and analyzing asymmetric scenarios arising for example from nonuniform cache sizes are open questions.

\section{F. Sharper Approximations}

The upper and lower bounds on the memory-rate tradeoff derived in this paper are shown here to be within a factor 12 from each other. Sharpening this approximation guarantee is of interest. As mentioned, numerical simulations suggest that a more careful analysis should be able to decrease the gap to within a factor of 5. To go beyond that, better lower and upper bounds are needed, both of which we know 
from Example 4 can be improved (see also the Appendix). Some further questions in this context are as follows.

- Linear versus Nonlinear Coding: As pointed out above, nonlinear coding schemes can offer unbounded gain in network and index coding [14], whereas for the caching problem linear coding schemes are sufficient to achieve optimality within a constant factor. This raises the questions if we can improve the gap using nonlinear schemes or if there exist caching networks for which nonlinear schemes can offer unbounded gain.

- Larger Field Size: All operations in this paper are over the binary field. In contrast, in network and index coding larger field sizes are useful. The question is thus if larger field sizes can improve the performance of coded caching.

- Coded Content Placement: Example 4 presents a scenario with two users, two files, and cache size $1 / 2$, where coded content placement can improve the achievable rate. While this type of coded content placement is not needed for a constant-factor approximation of the memory-rate tradeoff, it might lead to a smaller constant factor.

- Zero Error versus Vanishing Error: The problem setting as described in Section II allows for a vanishing error probability. However, the proposed achievable scheme has zero error probability, while still being approximately optimal. Schemes with vanishing error probability or lower bounds making explicit use of the zero-error requirement might be used to find sharper approximations.

\section{G. Implementation Complexity}

Compared to uncoded caching, the coded caching approach suggested in this paper imposes additional computational burden on the server and the users, especially for large values of $K$. One approach to deal with this burden is to use coded caching only among smaller subgroups of users. This results in lower computational load at the expense of higher rates over the shared link. Deriving the fundamental tradeoff between rate and memory subject to complexity constraints is of great interest.

\section{APPENDIX}

Example 4 in Section IV derives upper and lower bounds on the memory-rate tradeoff $R^{\star}(M)$ claimed in Theorems 1 and 2 for $K=N=2$. While these two bounds are sufficient to characterize $R^{\star}(M)$ to within a constant multiplicative gap, as we show in what follows, neither of the two bounds are tight in general.

We start by arguing that the achievable scheme can be improved by focusing on the case $M=1 / 2$. As before, we split each file into two subfiles, i.e., $A=\left(A_{1}, A_{2}\right)$ and $B=\left(B_{1}, B_{2}\right)$. In the placement phase, we choose the cache contents as $Z_{1}=A_{1} \oplus B_{1}$ and $Z_{2}=A_{2} \oplus B_{2}$. Assume that user one requests file $A$ and user two requests file $B$. The server can satisfy these requests by transmitting $\left(B_{1}, A_{2}\right)$ at rate $R=1$. The other three possible requests can be satisfied in a similar manner. This shows that $(M, R)$ pair $(1 / 2,1)$ is achievable. This new point improves the boundary of the achievable region from the solid blue to the dotted black curve in Fig. 3 in Section IV.

We now argue that the lower bound on can also be improved. We have for any achievable memory-rate pair $(M, R)$,

$$
\begin{aligned}
2 R F+2 M F & \geq H\left(X_{(1,2)}, Z_{1}\right)+H\left(X_{(2,1)}, Z_{2}\right) \\
& =H\left(X_{(1,2)}, Z_{1} \mid W_{1}\right)+H\left(X_{(2,1)}, Z_{2} \mid W_{1}\right)+I\left(W_{1} ; X_{(1,2)}, Z_{1}\right)+I\left(W_{1} ; X_{(2,1)}, Z_{2}\right) \\
& \geq H\left(X_{(1,2)}, Z_{1}, X_{(2,1)}, Z_{2} \mid W_{1}\right)+I\left(W_{1} ; X_{(1,2)}, Z_{1}\right)+I\left(W_{1} ; X_{(2,1)}, Z_{2}\right) \\
& \geq I\left(W_{2} ; X_{(1,2)}, Z_{1}, X_{(2,1)}, Z_{2} \mid W_{1}\right)+I\left(W_{1} ; X_{(1,2)}, Z_{1}\right)+I\left(W_{1} ; X_{(2,1)}, Z_{2}\right) .
\end{aligned}
$$


Now, since $W_{1}$ can be decoded from $\left(X_{(1,2)}, Z_{1}\right)$ and also from $\left(X_{(2,1)}, Z_{2}\right)$, and since $W_{2}$ can be decoded from $\left(X_{(1,2)}, Z_{1}, X_{(2,1)}, Z_{2}\right)$, we obtain using Fano's inequality that

$$
\begin{aligned}
I\left(W_{1} ; X_{(1,2)}, Z_{1}\right) & \geq F-\varepsilon F, \\
I\left(W_{1} ; X_{(2,1)}, Z_{2}\right) & \geq F-\varepsilon F, \\
I\left(W_{2} ; X_{(1,2)}, Z_{1}, X_{(2,1)}, Z_{2} \mid W_{1}\right) & \geq F-\varepsilon F
\end{aligned}
$$

for any $\varepsilon>0$ as long as $F$ is large enough. Hence,

$$
2 R F+2 M F \geq 3 F-3 \varepsilon F .
$$

Since $\varepsilon>0$ is arbitrary, this shows that

$$
R \geq 3 / 2-M
$$

Since this is true for any achievable $(M, R)$ pair, this implies that

$$
R^{\star}(M) \geq 3 / 2-M \text {. }
$$

This bound, together with the two cut-set bounds derived earlier, proves that the dotted black curve depicted in Fig. 3 in Section IV is indeed equal to $R^{\star}(M)$.

\section{ACKNOWLEDGMENT}

The authors would like to thank S. Borst and S. Kennedy for their comments on an earlier draft of this paper as well as the reviewers for their careful reading of the manuscript.

\section{REFERENCES}

[1] L. W. Dowdy and D. V. Foster, "Comparative models of the file assignment problem," ACM Comput. Surv., vol. 14, pp. 287-313, June 1982.

[2] K. C. Almeroth and M. H. Ammar, "The use of multicast delivery to provide a scalable and interactive video-on-demand service," IEEE J. Sel. Areas Commun., vol. 14, pp. 1110-1122, Aug. 1996.

[3] A. Dan, D. Sitaram, and P. Shahabuddin, "Dynamic batching policies for an on-demand video server," Multimedia Syst., vol. 4, pp. 112-121, June 1996.

[4] M. R. Korupolu, C. G. Plaxton, and R. Rajaraman, "Placement algorithms for hierarchical cooperative caching," in Proc. ACM-SIAM SODA, pp. 586-595, Jan. 1999.

[5] A. Meyerson, K. Munagala, and S. Plotkin, "Web caching using access statistics," in Proc. ACM-SIAM SODA, pp. 354-363, 2001.

[6] I. Baev, R. Rajaraman, and C. Swamy, "Approximation algorithms for data placement problems," SIAM J. Comput., vol. 38, pp. 14111429 , July 2008.

[7] S. Borst, V. Gupta, and A. Walid, "Distributed caching algorithms for content distribution networks," in Proc. IEEE INFOCOM, pp. 1478-1486, Mar. 2010.

[8] Y. Birk and T. Kol, "Coding on demand by an informed source (ISCOD) for efficient broadcast of different supplemental data to caching clients," IEEE Trans. Inf. Theory, vol. 52, pp. 2825-2830, June 2006.

[9] Z. Bar-Yossef, Y. Birk, T. S. Jayram, and T. Kol, "Index coding with side information," IEEE Trans. Inf. Theory, vol. 57, pp. 1479-1494, Mar. 2011.

[10] T. M. Cover and J. A. Thomas, Elements of Information Theory. Wiley, 1991.

[11] M. Effros, S. El Rouayheb, and M. Langberg, "An equivalence between network coding and index coding," arXiv:1211.6660 [cs.IT], Nov. 2012.

[12] R. Ahlswede, N. Cai, S.-Y. R. Li, and R. W. Yeung, "Network information flow," IEEE Trans. Inf. Theory, vol. 46, pp. 1204-1216, Apr. 2000.

[13] M. Langberg and A. Sprintson, "On the hardness of approximating the network coding capacity," IEEE Trans. Inf. Theory, vol. 57, pp. 1008-1014, Feb. 2011.

[14] A. Blasiak, R. Kleinberg, and E. Lubetzky, "Lexicographic products and the power of non-linear network coding," in Proc. IEEE FOCS, Oct. 2011.

[15] M. A. Maddah-Ali and U. Niesen, "Decentralized coded caching attains order-optimal memory-rate tradeoff," arXiv:1301.5848 [cs.IT], Jan. 2013.

[16] D. D. Sleator and R. E. Tarjan, "Amortized efficiency of list update and paging rules," Communications ACM, vol. 28, pp. 202-208, Feb. 1985.

[17] R. Pedarsani, M. A. Maddah-Ali, and U. Niesen, "Online coded caching," arXiv:1311.3646 [cs.IT], Nov. 2013.

[18] U. Niesen and M. A. Maddah-Ali, "Coded caching with nonuniform demands," arXiv:1308.0178 [cs.IT], Aug. 2013.

[19] M. Ji, G. Caire, and A. Molisch, "Fundamental limits of distributed caching in D2D wireless networks," in Proc. IEEE ITW, Sept. 2013. 